\title{
The Kinetics of Joined Action of Triplet-Triplet Annihilation and First-Order Decay of Molecules in the $T_{1}$ State in the Case of Nondominant First-Order Process
}

\author{
Pawel Borowicz ${ }^{1,2,3}$ and Bernhard Nickel ${ }^{1}$ \\ ${ }^{1}$ Spectroscopy and Photochemical Kinetics Department, Max-Planck-Institut Für biophysikalische Chemie, \\ Am Fassberg 11, 37077 Göttingen, Germany \\ ${ }^{2}$ Institute of Physical Chemistry, Polish Academy of Sciences, Kasprzaka 44/52, 01-224 Warsaw, Poland \\ ${ }^{3}$ Department of Characterisation of Nanoelectronic Structures, Institute of Electron Technology, Al. Lotników 32/46, \\ 02-668 Warsaw, Poland
}

Correspondence should be addressed to Paweł Borowicz, borowicz@ite.waw.pl

Received 9 April 2012; Accepted 28 May 2012

Academic Editors: W. A. Badawy and S.-I. Kondo

Copyright () 2012 P. Borowicz and B. Nickel. This is an open access article distributed under the Creative Commons Attribution License, which permits unrestricted use, distribution, and reproduction in any medium, provided the original work is properly cited.

This paper presents the description of triplet-triplet annihilation in the case of nondominant first-order decay of molecules in the triplet state. The kinetics of the statistical system is influenced by joined action of two processes: the first- and second-order decies. This kinetics can be described with analytical function if the rate parameter of second-order reaction is constant. The approach presented here combines the well-known Smoluchowski formula with the previously published intuitive and non-Fickian models of diffusion-controlled triplet-triplet annihilation. The kinetics of the delayed fluorescence of anthracene is used as a practical example of applicability of the model proposed. The advantages and limits of the proposed model are discussed.

\section{Introduction}

The reaction where the diffusion plays an important role was studied for many years [1]. Nowadays studied diffusioncontrolled reactions like fluorescence quenching [2] very often take place on the picosecond timescale. Although the Smoluchowski-Collins-Kimball (SCK) model shows some difficulties in reproduction of the kinetic parameters, this approach or its variations are used for the interpretation of the kinetic data. An example of the limits of the SCK model in the description of kinetics of fluorescence quenching is the treatment of the solvent as continuous medium. This approximation seems not to be valid on the picosecond timescale [3]. The other inaccuracy arising from the continuous-medium approximation is reflected in the value of the effective annihilation radius. The effect of the screening by the first coordination shell gives this value $20 \%$ to $40 \%$ larger than the value calculated from dimensions of the molecule [4]. The other example of more complicated kinetics than that predicted by the SCK model is the intermolecular charge transfer $(\mathrm{CT})[5,6]$. In this case the important role is played not only by the translational diffusion of the molecules but the rotational one as well. The rotational diffusion influences the mutual orientation of the molecules. And the mutual orientation plays an important role in the efficiency of charge-transfer reaction. The authors of $[5,6]$ used three exponential phenomenological models for the description of the kinetics of the system with nonnegligible rotational diffusion. The diffusion also plays an important role in the kinetics of intermolecular protontransfer (PT) reaction [7-15]. For example, in the case of PT from 5-cyano-2 naphthol in methanol/water mixture the 
nonexponential kinetics is observed [7]. The kinetics of the diffusion-influenced PT is generally described by DebyeSmoluchowski-Einstein (DSE) equation. The most general description is realized with the numerical solution of the DSE equation [11-13]. In some cases analytical or approximate solutions of DSE equation also offer reasonable description of the PT kinetics $[12,13]$. In the case of large delay the concentration of the reacting species is so low that the diffusion plays an important role. In such conditions the decay of the fluorescence should be described by power function instead of the exponential one $[9,10]$, for example in the case of derivatives of 1- and 2-naphthol the asymptotic decay of the reacting species can be described with power law $\sim t^{-3 / 2}$ [8]. There are however deviations from the power law $\sim t^{3 / 2}$ in the case of some diffusion-controlled reactions, for example, due to finite rate of recombination within the contact pair in acid-base systems [16]. In such systems the internal rate parameter of the proton transfer reaction can be measured for high concentration of reacting species where the diffusion does not play the most important role [16].

In the case of triplet-triplet annihilation (TTA) in viscous solvents where the process takes place on microsecond timescale the deviations from diffusional Smoluchowski model [17] are not observed directly [18]. It means that the course of the intensity of delayed fluorescence as a function of time is well described by the function derived from Smoluchowski approach. The deviations from this model manifest themselves more subtle. For example, the effect of screening by the first coordination shell [4] can be detected as larger value of annihilation radius than that calculated from molecular dimensions [19]. Also partly reflecting boundaries [20] should give no important contribution, because the movement of the molecules in viscous solvent is very slow. The mutual diffusion coefficient of molecules in typical diffusion-controlled TTA experiment is at least three orders of magnitude lower than the typical diffusion coefficient of organic molecules in organic solvents $\left(10^{-9} \mathrm{~m}^{2} \mathrm{~s}^{-1}\right)$ at room temperature. The Smoluchowski model can be applied to describe the kinetics of statistical system with partly reflecting boundaries. However, instead of the geometrical radius of interaction the effective one has to be used [21, pages (105-117)]. The effective radius of interaction is larger in comparison with the parameter used originally by Smoluchowski. For typical organic molecule in organic solvent at room temperature the effective radius in Smoluchowski approach used to describe the system with partly reflecting boundaries should be $10 \%$ larger than the geometrical one. This difference between "real" and "effective" radius of interaction is difficult to be detected in the experiment [21, pages (105-117)]. So in the case of TTA the assumptions of the original Smoluchowski approach seems to be a good approximation: the probability of the reaction for molecules within annihilation radius equals $100 \%$ (the reaction takes place immediately). With other words: due to high viscosity of the solvent in TTA experiment the molecules which build the contact pair cannot diffuse away, because they stay in the contact pair long enough to neglect the influence of the internal rate parameter on the kinetics of the whole process.
Also the reacting species can be treated as spherically symmetric. There are two reasons that make this approximation valuable. The first one is the very fast rotation of the molecules in comparison with their translational diffusion. This aspect was discussed in earlier papers [19, 22]. In general case the translational and rotational diffusion should be treated simultaneously. However, very often the correction for rotational diffusion results in a constant factor multiplying the time-dependent rate parameter obtained from the second Fick's law [23, pages (25-26)]. This kind of correction is automatically included in the amplitude of fitted function which describes the kinetics of the process.

The complicated spatial feature of Dexter mechanism of interaction [24] is not taken into account. Due to its very small spatial range of interaction the influence on the kinetics of the process can be treated as negligible. The influence of the spin statistics on the kinetics of TTA was referred to in previous papers $[19,22]$. Also the existence of different TTA channels and their contribution to the kinetics of the TTA was summarized there.

To sum up, in the description of TTA kinetics of in viscous solutions the molecules can be treated as hard spheres which react with each other only when they are in the contact.

In order to make the discussion of the joined action of first-order decay and triplet-triplet annihilation the description is done within basic assumption of the classical Smoluchowki [17] model-mathematically simplest possible treatment of the problem:

(i) the Brownian motions of the reacting particles can be described by diffusion in continuous medium;

(ii) the molecules at distances larger than annihilation radius do not interact with each other;

(iii) the annihilation within contact pair takes place immediately, it means that the molecules in the $\mathrm{T}_{1}$ state cannot diffuse away if they are within an annihilation radius from each other;

(iv) the evolution of the local density $\rho(r, t)$ starts from random distribution of the particles in solution $\rho(r, t=0)=\rho_{0}$ and tends to $\rho(r, t \rightarrow \infty)$.

The summarized above assumptions led Smoluchowski to his famous formula for the time-dependent rate parameter $k_{2 A}(t)$ :

$$
k_{2 A}(t)=4 \pi R_{A} D_{T}\left(1+\frac{R_{A}}{\sqrt{\pi D_{T} t}}\right),
$$

where $R_{A}$ is the annihilation radius and $D_{T}$ is the mutual diffusion coefficient. However, the original Smoluchowski model does not take into account the joined action of two processes: the first-order decay of the molecules in the $T_{1}$ state and the diffusion-controlled TTA. So the correction has to be introduced. In previous papers $[19,22]$ we concentrated on the case of strongly dominant first-order decay. That condition allowed to introduce the following approximation: the decay of the average density of molecules in the $T_{1}$ state was described by monoexponential function. 
The Smoluchowski solution of the second Fick's law was modified in order to introduce the joined action of both processes: the first-order decay and the spatial redistribution, to the expression for time-dependent rate parameter. In the intuitive model the term $\exp \left(-k_{T} t\right)$ was introduced to the denominator of so-called "short-time" part of $k_{2 A}(t)$ [22]. The other approach was presented in [19] where the time-dependent mutual diffusion coefficient in the form $D_{T} \exp \left(-2 k_{T} t\right)$ was used in the second Fick's law instead of the constant diffusion coefficient $D_{T}$. The experimental conditions of the measurements that allowed to test the models were very unfavorable. Generally the $p$-type delayed fluorescence is a few orders of magnitude smaller than the prompt one. In order to obtain good signal-to-noise ratio the measurement is time consuming (the measurement of a single decay took over 10 hours). The investigation of the diffusion-controlled TTA kinetics requires also extremely high stability of the temperature: it must be constant within the range of $\pm 0.01 \mathrm{~K}$. The reason for this extremely high stability is to keep the viscosity of the medium constant within a few percent.

In this paper we extend the modifications of the Smoluchowki time-dependent rate parameter to the case of nondominant first-order decay. The modified models (two variations) are applied to the description of the TTA kinetics of anthracene in the mixture of cis- and transdimethylcyclohexane (cis/trans-DMCH).

\section{Description of the Model}

Let us come back to the analysis of the TTA presented in paper [22]. The reason to use the time-dependent rate parameter $k_{2 A}(t)$ in the form:

$$
k_{2 A}(t)=4 \pi R_{A} D_{T}\left(1+\frac{R_{A}}{e^{-K_{T} t} \sqrt{\pi D_{T} t}}\right),
$$

was to introduce the contribution of the first-order decay to the evolution of the local density. Since in that case the first-order reaction was dominant the joined action of two processes, first-order decay and diffusion-controlled TTA, was described by introducing the exponential function in the denominator of component representing so-called "shorttime" effect in (1). This led to (2).

In order to extend the intuitive model presented in paper [22] to the case of nondominant first-order decay we carry out similar reasoning as in that paper.

Let us take into account three molecules in the $T_{1}$ state and denote them with letters $A, B$, and $C$. Let us look at the kinetics of the system from the point of view of $A$. The molecule $A$ can react with one of two others $(B$ or $C$ ) if the reaction partner is still in the $T_{1}$ state when molecules come to the contact distance. Each of the potential reaction partner for $A$ can decay on the first-order reaction before building the contact pair with $A$. It is also possible that both $B$ and $C$ decay due to diffusion-controlled TTA:

$$
\begin{aligned}
{ }^{3} B^{*}+{ }^{3} C^{*} & \longrightarrow{ }^{1}(B C){ }^{* *} \longrightarrow{ }^{1} B^{*}+{ }^{1} C\left(\text { or }{ }^{1} B+{ }^{1} C^{*}\right) \\
& \longrightarrow{ }^{1} B+{ }^{1} C+h v
\end{aligned}
$$

where ${ }^{3} B^{*}$ and ${ }^{3} C^{*}$ denote the molecules in the $T_{1}$ state, ${ }^{1}(B C)^{* *}$ denotes the encounter pair with singlet multiplicity, ${ }^{1} B^{*}$ and ${ }^{1} C^{*}$ are the molecules in the $S_{1}$ state, and ${ }^{1} B$ and ${ }^{1} C$ are in the ground state; $h v$ denotes the energy of the emitted photon. Following the same course of reasoning as in previous paper [22] that the kinetics of TTA should be determined by diffusion of molecules in the $T_{1}$ state which is in turn influenced by the decay of the average density of molecules in the triplet state, one can propose the following description of the kinetics. Since the main influence of the joined action can be limited to the "so-called" shorttime effect [25] one can approximate the description of the kinetics in the following way:

(1) the stationary part of the standard Smoluchowski rate parameter $k_{2 A}(t)$ remains unchanged;

(2) the time-dependent part $R_{A} /\left(\pi D_{T} t\right)^{1 / 2}$ is modified in such a way that takes into account the influence of the decay of the average density on the total amount of diffusing molecules.

The denominator in the short-time part of standard Smoluchowski parameter $k_{2 A}(t)$ is proportional to the total number of diffusing molecules within the period from $t=0$ to $t>0$ under the assumption, that there are no other processes of decay of molecules. In the case of TTA the amount of diffusing molecules will be decreased due to the decay of the average density. The total amount of molecules which diffuse during the period from $t=0$ to $t>0$ should be proportional to the $\left(\rho(t) / \rho_{0}\right)\left(\pi D_{T} t\right)^{1 / 2}$. Finally the intuitive expression for time-dependent rate parameter should have the form:

$$
k_{2 A}(t)=4 \pi R_{A} D_{T}\left(1+\frac{R_{A}}{\left(\rho(t) / \rho_{0}\right) \sqrt{\pi D_{T} t}}\right) .
$$

Now one has to find an expression for $\rho(t)$. The start point is the well-known formula for the concentration $\rho(t)$ of molecules in the triplet state obtained in the case of timeindependent rate constant of the TTA. The expression can be found elsewhere [26]:

$$
\rho(t)=\rho_{0} \frac{e^{-k_{T} t}}{1+k_{T T}\left(\rho_{0} / k_{T}\right)\left(1-e^{-K_{T} t}\right)},
$$

where $\rho_{0}$ is the initial concentration of the molecule in the $T_{1}$ state, $k_{T}$ is the first-order rate constant, and $k_{T T}$ is the second-order rate constant. Since in our case the TTA is diffusion controlled we approximate the $\rho(t)$ by replacing $k_{T T}$ with $k_{2 A}(t)$. The expression for average density has the following form:

$$
\begin{aligned}
& \rho(t) \\
& =\rho_{0} \frac{e^{-k_{T} t}}{1+\left(k_{2 A}(t) \rho_{0} / k_{T}\right)\left(1-e^{-k_{T} t}\right)} \\
& =\rho_{0} \frac{e^{-k_{T} t}}{1+4 \pi R_{A} D_{T}\left(\rho_{0} / k_{T}\right)\left(1+R_{A} /\left(\rho(t) / \rho_{0}\right) \sqrt{\pi D_{T} t}\right)\left(1-e^{-k_{T} t}\right)} .
\end{aligned}
$$


Equation (6) can be resolved due to the average density $\rho(t)$ :

$$
\rho(t)=\rho_{0} \frac{e^{-k_{T} t}-4 \sqrt{\pi D_{T}} R_{A}^{2}\left(\rho_{0} / k_{T} \sqrt{t}\right)\left(1-e^{-k_{T} t}\right)}{1+4 \pi D_{T} R_{A}\left(\rho_{0} / K_{T}\right)\left(1-e^{-k_{T} t}\right)} .
$$

Substituting the ratio $\rho(t) / \rho_{0}$ from (7) to (4) one obtains:

$$
k_{2 A}(t)=4 \pi R_{A} D_{T}\left(1+\frac{R_{A}}{\left(\left(e^{-k_{T} t}-4 \sqrt{\pi D_{T}} R_{A}^{2}\left(\rho_{0} / K_{T} \sqrt{t}\right)\left(1-e^{-k_{T} t}\right)\right) /\left(1+4 \pi D_{T} R_{A}\left(\rho_{0} / k_{T}\right)\left(1-e^{-k_{T} t}\right)\right)\right) \sqrt{\pi D_{T} t}}\right) .
$$

The expression for the time-dependent intensity of the delayed fluorescence has the form [27]:

$$
I_{D F}(t)=C k_{2 A}(t) \rho(t)^{2}
$$

where $C$ is constant and $k_{2 A}(t)$ is time-dependent rate parameter. Introducing the average density $\rho(t)$ is as in (7) and $k_{2 A}(t)$ from (8) one obtains the following equation for the intensity of the delayed fluorescence:

$$
\begin{aligned}
& I_{D F}(t) \\
& =C 4 \pi R_{A} D_{T}\left(1+\frac{R_{A}}{\frac{e^{-k_{T} t}-\frac{4 \sqrt{\pi D_{T}} R_{A}^{2} \rho_{0}}{k_{T} \sqrt{t}}\left(1-e^{-k_{T} t}\right)}{1+\frac{4 \pi R_{A} D_{T} \rho_{0}}{k_{T}}\left(1-e^{-k_{T} t}\right)} \sqrt{\pi D_{T} t}}\right) \\
& \times\left(\rho_{0} \frac{e^{-k_{T} t}-\frac{4 \sqrt{\pi D_{T}} R_{A}^{2} \rho_{0}}{k_{T} \sqrt{t}}\left(1-e^{-k_{T} t}\right)}{1+\frac{4 \pi R_{A} D_{T} \rho_{0}}{k_{T}}\left(1-e^{-k_{T} t}\right)}\right)^{2} .
\end{aligned}
$$

The above presented formula can be recognized as an extension of the time-dependent rate parameter $k_{2 A}(t)(2)$. Instead of $\exp \left(-k_{T} t\right)$ in the case of the dominant first-order decay the ratio $\rho(t) / \rho_{0}$ is introduced in the denominator of the short-time term. If the second term in the nominator in (7) is negligible:

$$
\frac{4 \sqrt{\pi D_{T}} R_{A}^{2} \rho_{0}}{k_{T} \sqrt{t}} \approx 0
$$

Equation (10) can be reduced to the following form:

$$
\begin{aligned}
& I_{D F}(t) \\
& =C 4 \pi R_{A} D_{T}\left(1+\frac{R_{A}}{\frac{e^{-k_{T} t}}{1+\frac{4 \pi R_{A} D_{T} \rho_{0}}{k_{T}}\left(1-e^{-k_{T} t}\right)} \sqrt{\pi D_{T} t}}\right) \\
& \\
& \times\left(\frac{\rho_{0} e^{-k_{T} t}}{1+\frac{4 \pi R_{A} D_{T} \rho_{0}}{k_{T}}\left(1-e^{-k_{T} t}\right)}\right)^{2} .
\end{aligned}
$$

If the second term in the denominator in (7) is also negligible:

$$
\frac{4 \cdot \pi D_{T} R_{A} \rho_{0}}{k_{T}} \approx 0 .
$$

Equation (10) is reduced to the form discussed in the paper [22]:

$$
I_{D F}(t)=C 4 \pi R_{A} D_{T}\left(1+\frac{R_{A}}{e^{-k_{T} t} \sqrt{\pi D_{T} t}}\right) e^{-2 k_{T} t}
$$

Non-Fickian Effects in the Nonexponential Decay. In order to compare the intuitive model [22] with the non-Fickian one [19] we decided to introduce another type of the modification of $I_{D F}(t)$. The starting points are (5) with $k_{T T}=$ $k_{2 A}(t)$ and (9). Now we assume that the nonexponential behavior influence the average density $\rho(t)$, but in turn the local density can be reasonably described with the exponential contribution of the first-order decay to the redistribution process. It means that TTA can contribute to the behavior of the average concentration of the molecules in the $T_{1}$ state. At the other side the correction to the average density is so small that the time-dependent rate parameter $k_{2 A}(t)$ can be reasonably described for the dominant firstorder decay. This kind of approximation can be treated as a second step after that presented in papers [19] and [22]. In those papers the first-order decay influenced redistribution but the opposite influence was negligible. Now we introduce nonnegligible contribution of the TTA to the average density. This contribution is however so small that it is not recognized 


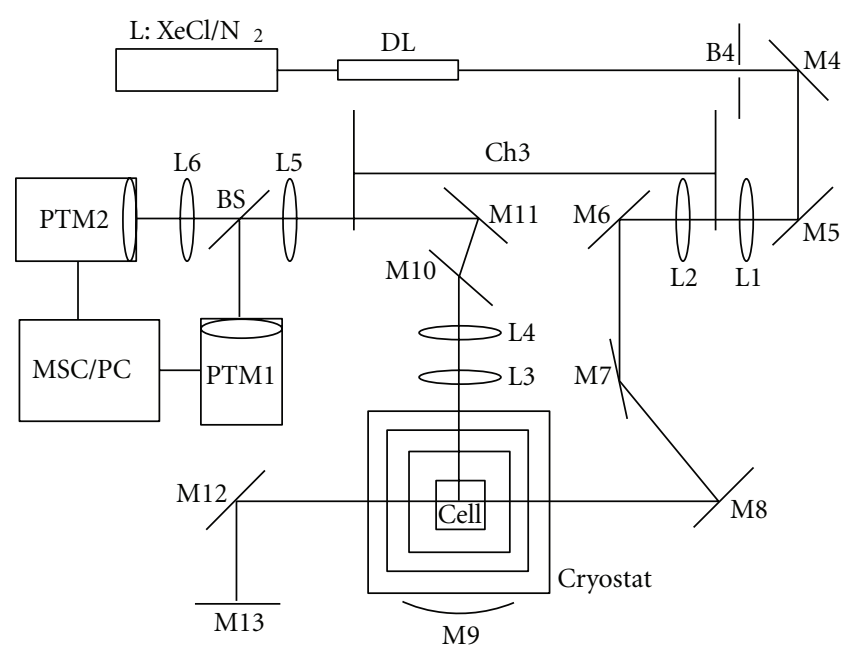

FIGURE 1: Modification of the previously described [19, 22] experimental setup for excitation with pulsed lasers. in the time-dependent rate parameter $k_{2 A}(t)$. This lead to the following form of the nonexponential model for the $I_{D F}(t)$ :

$$
I_{D F}(t)=C k_{2 A}(t)\left(\frac{e^{-k_{T} t}}{1+\left(k_{2 A}(t) / k_{T}\right)\left(1-e^{-k_{T} t}\right)}\right)^{2}
$$

where $k_{2 A}(t)$ can be taken from (2) - intuitive version [22] or from paper [19]—non-Fickian version:

$$
\begin{aligned}
I_{D F}(t)= & C 4 \pi D_{T} R_{A}\left(1+\frac{R_{A}}{e^{-t_{T} t} \sqrt{\pi D_{T} t}}\right) \\
& \times\left(\frac{e^{-k_{T} t}}{1+\left(4 \pi D_{T} R_{A} \rho_{0} / k_{T}\right)\left(1+R_{A} / e^{-k_{T} t} \sqrt{\pi D_{T} t}\right)\left(1-e^{-2 k_{T} t}\right)}\right)^{2}, \\
I_{D F}(t)= & C 4 \pi D_{T} R_{A}\left(1+\frac{\sqrt{2 k_{T}} R_{A}}{\sqrt{\pi D_{T}\left(1-e^{-2 k_{T} t}\right)}}\right) \\
& \times\left(\frac{e^{-k_{T} t}}{1+\left(4 \pi D_{T} R_{A} \rho_{0} / k_{T}\right)\left(1+\sqrt{2 k_{T}} R_{A} / \sqrt{\pi D_{T}\left(1-e^{-2 k_{T} t}\right)}\right)\left(1-e^{-k_{T} t}\right)}\right)^{2} .
\end{aligned}
$$

\section{Experimental}

The purification of anthracene and the components of mixture solvent, as well as the preparation of the sample were described in previous papers [19,22].

The experimental setup used for these experiments was similar to that described previously $[19,22]$. The only one modification was the excitation source. Instead of $\mathrm{cw}$ $\mathrm{Ar}^{+}$laser and two slow choppers pulsed laser was used. The excitation wavelength was equal to $363.3 \mathrm{~nm}$. Two configurations of excitation source were used:

(1) dye laser pumped $\mathrm{N}_{2}$ laser;

(2) dye laser (Lambda Physik LPD 3000) pumped excimer laser (Lambda Physik LPX 100).

The first option of excitation will be called "short excitation pulse" (the temporal half width of the pulse $\sim$ $1 \mathrm{~ns}$ ), the other one "middle excitation pulse" (the temporal half width of the pulse $\sim 20 \mathrm{~ns}$ ). The laser pulses were focused on the first disc of the fast chopper as presented in Figure 1. The measurements of the delayed fluorescence and phosphorescence were done as in the case of excitation with the "long pulse" [19, 22].
The decays were measured in the temperature range from $132 \mathrm{~K}$ to $152 \mathrm{~K}$ in the case of middle excitation pulse and in the range $132 \mathrm{~K}-150 \mathrm{~K}$ for the short pulse. The increment between the temperature values was equal to $1 \mathrm{~K}$.

3.1. Evaluation Procedure. In order to make our model mathematically as simple as possible we use here similar procedure as in the previous papers $[19,22]$. We do not use the modified (Nickel's) initial conditions. The reasons are the same as reported previously: these initial conditions affect only the initial part of the decay (about $1 \mathrm{~ms}$ ) leaving the main part of the decay unchanged. We use terms antiSmoluchowski time range and Smoluchowski time range in the same meaning as previously:

(i) anti-Smoluchowski time range: the part of a decay where the kinetics significantly depends on the type of initial conditions;

(ii) Smoluchowski time range: the part of a decay where the kinetics is practically independent of the type of initial conditions. 
The main attention is focused on the period between the end of the anti-Smoluchowski time range and the final instant of the nonstationary part of the $k_{2 A}(t)$. In this time range the nonstationary part of the kinetics has a significant contribution to the total kinetics of the sample. This contribution offers the possibility to obtain the parameter like annihilation radius.

The delayed fluorescence was analyzed with the following functions:

$$
\begin{aligned}
& I_{D F}(t)=P_{1}\left(1+\frac{P_{2}}{e^{-t / P_{3}}-\frac{P_{2} P_{4}\left(1-e^{-t / P_{3}}\right)}{\frac{\sqrt{t}}{1+P_{4}\left(1-e^{-t / P_{3}}\right)} \sqrt{t}}}\right) \\
& \times\left(\frac{e^{-t / P_{3}}-\frac{P_{2} P_{4}\left(1-e^{-t / P_{3}}\right)}{\sqrt{t}}}{1+P_{4}\left(1-e^{-t / P_{3}}\right)}\right)^{2}+P_{0} \\
& I_{D F}(t)=P_{1}\left(1+\frac{P_{2}}{\frac{e^{-t / P_{3}}}{1+P_{4}\left(1-e^{-t / P_{3}}\right)} \sqrt{t}}\right) \\
& \times\left(\frac{e^{-t / P_{3}}}{1+P_{4}\left(1-e^{-t / P_{3}}\right)}\right)^{2}+P_{0},
\end{aligned}
$$

where $P_{0}$ is the dark current, $P_{1}$ is the amplitude, $P_{2}=$ $R_{A} /\left(\pi D_{T}\right)^{1 / 2}$ is the parameter that describes short-time effect, $P_{3}=1 / k_{T}$ is the decay time of the molecules via first-order reaction, and $P_{4}=4 \pi R_{A} D_{T} \rho_{0} / k_{T}$ is the ratio of original Smoluchowski rate parameter in the case of stationary diffusion $4 \pi R_{A} D_{T}$ multiplied with the initial concentration of molecules in the $T_{1}$ state $\rho_{0}$ and divided through the rate parameter of the first-order decay $k_{T} . P_{4}$ will be called the indicator of nonexponential behavior. The condition $P_{4} \leq 0.01$ was used by Nickel and others [18, 27] as a limit of dominant first-order decay in TTA kinetics.

The parameters obtained from nonexponential decay were compared with the parameters obtained from model described in [22]:

$$
I_{D F}(t)=P_{1}\left(1+\frac{P_{2}}{e^{-t / P_{3}} \sqrt{t}}\right) e^{-2 t / P_{3}}+P_{0} .
$$

The functions used to compare the nonexponential versions of the intuitive (20) and the non-Fickian (21) models have the following form:

$$
\begin{aligned}
I_{D F}(t) & \\
= & P_{1}\left(1+\frac{P_{2}}{e^{-t / P_{3}} \sqrt{t}}\right) \\
& \times\left(\frac{e^{-t / P_{3}}}{1+P_{4}\left(1+\left(P_{2} / e^{-t / P_{3}} \sqrt{t}\right)\right)\left(1-e^{-t / P_{3}}\right)}\right)^{2}+P_{0},
\end{aligned}
$$

$$
\begin{aligned}
& I_{D F}^{M}(t) \\
& =P_{1}\left(1+\sqrt{2 / \pi}\left(P_{2} / \sqrt{P_{3}} \sqrt{1-e^{-2 \mathrm{t} / P_{3}}}\right)\right) \\
& \quad \times\left(\frac{e^{-t / P_{3}}}{1+P_{4}\left(1+\sqrt{2 / \pi}\left(P_{2} / \sqrt{P_{3}} \sqrt{1-e^{-2 \mathrm{t} / P_{3}}}\right)\right)\left(1-e^{-t / P_{3}}\right)}\right)^{2} \\
& \quad+P_{0} .
\end{aligned}
$$

As in the previous papers $[19,22]$ the points from the begining of the decay curve were systematically cut off with the increment of 10 points. The parameters obtained from fitting procedure were plotted as a function of the delay (starting point of evaluation). This procedure was also applied in the case of asymptotic function derived from (17) and (18) by neglecting the short-time term. From asymptotic functions it was obtained that the redistribution term has negligible contribution for the delay longer than the certain value. The time range where the short-time term significant contribution to the kinetics changes with the temperature (viscosity). The reason for such a behavior is the different mobility of the molecules. The value of $10 \mathrm{~ms}$ is the upper limit of the time range of particular interest. The results of fitting of asymptotic functions are generally close to the fit of exponential asymptotic function.

Phosphorescence decays were analyzed with the following functions:

$$
\begin{gathered}
I_{\mathrm{ph}}^{\text {non-exp. }}(t)=P_{1} \frac{e^{-t / P_{3}}}{1+P_{4}\left(1-e^{-t / P_{3}}\right)}+P_{0}, \\
I_{\mathrm{ph}}^{\exp }(t)=P_{1} e^{-t / P_{3}}+P_{0},
\end{gathered}
$$

where the signs $P_{0}, P_{1}, P_{3}$, and $P_{4}$ have the same meaning as in the case of the delayed fluorescence. Since the contribution of the short-time effect to the phosphorescence is much smaller in comparison with the delayed fluorescence the simplified version of the expression for the average density $\rho(t)$ in the nonexponential case was used. In this formula the term with the product $P_{2} P_{4}$ is avoided. In order to avoid small inaccuracies coming from short-time effect first few ms were removed from the decay prior to the fit.

\section{Results and Discussion}

In Figure 2 there is an example of the result of fitting procedure: the decay of the delayed fluorescence of anthracene measured at $146 \mathrm{~K}$, exited with the middle excitation pulse, presented in the plot parameters, obtained for nonexponential fit and are calculated from (17). Since results obtained with application of the simplified function defined by (18) have almost the same values they are omitted. The values of parameters obtained from nonexponential fit, are compared with the result of exponential approximation-(19). The values of kinetic parameters are presented as a function of the delay $\left(t_{0}\right)$. In Figure 2(a) there is presented the first-order rate constant of the molecules in the $T_{1}$ state: 


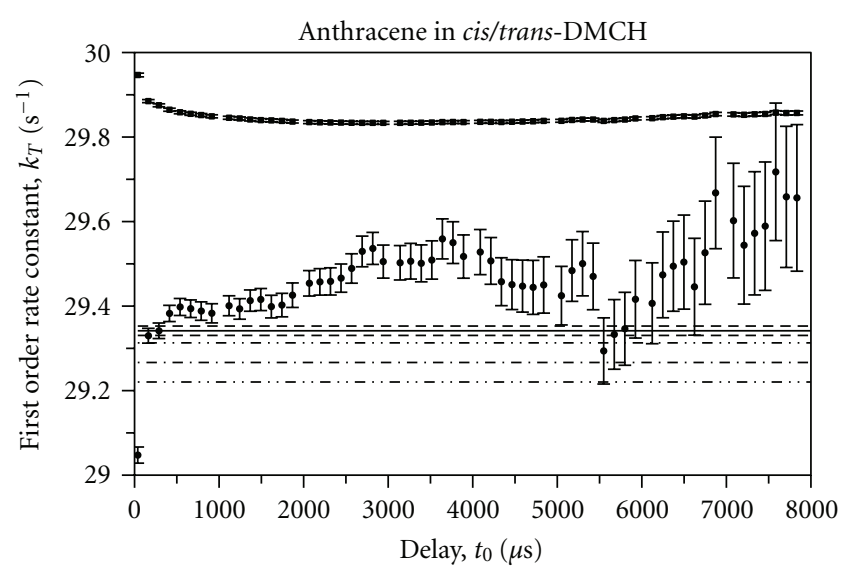

(a)

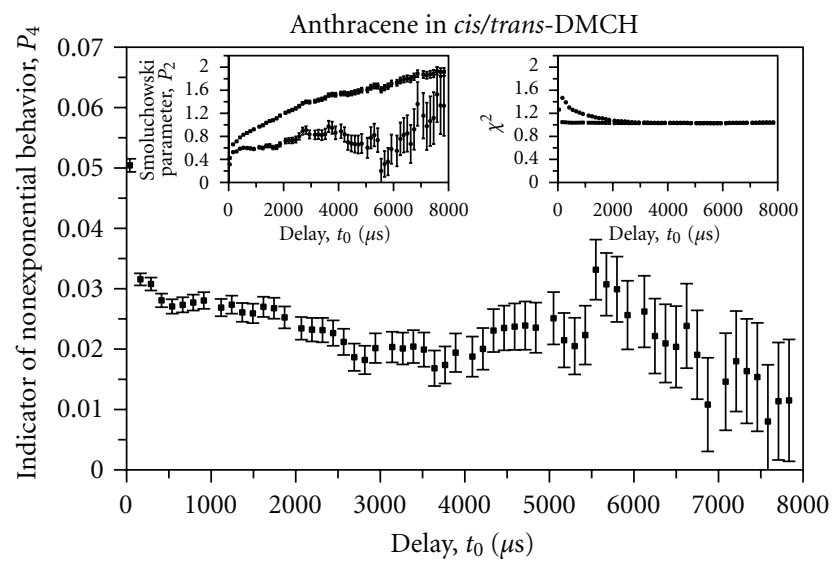

(b)

Figure 2: The fitted parameters as dependent on the delay (the starting point of evaluation $-t_{0}$ ) obtained for the delay of anthracene in cis/trans-DMCH at $146 \mathrm{~K}$ for middle excitation pulse. Part (a): first-order rate constant $k_{T}$ : squares $\left(k_{T}^{\mathrm{df}, \mathrm{e}}\right)$ —delayed fluorescence, exponential fit $(21)$; circles $\left(k_{T}^{\mathrm{dffne}}\right)$ —delayed fluorescence, nonexponential fit (17); solid line $\left(k_{T}^{\mathrm{ph}, \mathrm{e}}\right)$-phosphorescence, exponential fit; dashed lines-accuracy of $k_{T}^{\mathrm{ph}, \mathrm{e}}$; dash-dotted line $\left(k_{T}^{\mathrm{ph}, \mathrm{ne}}\right)$-phosphorescence, nonexponential fit; dash-dot-dotted lines-the accuracy of $\left(k_{T}^{\mathrm{ph}, \mathrm{ne}}\right)$. Part (b): main plot indicator of nonexponential $\left(P_{4}\right)$ behavior; right inset: comparison of smoluchowski parameter $\left(P_{2}\right)$ for nonexponential fit (lower curve) and exponential fit (upper curve); left inset: autocorrelation function for nonexponential fit (lower curve) and exponential fit (upper curve).

(i) squares-calculated from the delayed fluorescence with exponential model $\left(k_{T}^{\mathrm{df}, \mathrm{e}}\right)$;

(ii) circles-obtained from the delayed fluorescence within nonexponential approach $\left(k_{T}^{\mathrm{df}, n e}\right)$;

(iii) solid line-calculated from phosphorescence with exponential model $\left(k_{T}^{\mathrm{ph}, \mathrm{e}}\right)$;

(iv) dot-dashed line-calculated from phosphorescence with application of nonexponential model $\left(k_{T}^{\mathrm{ph}, \mathrm{ne}}\right)$.

The dashed lines present the standard deviations of the exponential model: $\Delta k_{T}^{\mathrm{ph}, \mathrm{e}}$ and dash-dot-dotted line marks the standard deviation of the nonexponential model: $\Delta k_{T}^{\mathrm{ph} \text {,ne }}$ in the case of the phosphorescence decay. In the case of exponential fit the calculated standard deviation is so small for the delayed fluorescence that it cannot be reproduced on the plot although the ordinate is expanded: the presented range is between $29 \mathrm{~s}^{-1}$ and $30 \mathrm{~s}^{-1}$. In the case of the exponential fit of the delayed fluorescence and for the delay time larger than about $1 \mathrm{~ms}$ the values of the first-order rate constant $k_{T}^{\mathrm{df}, \mathrm{e}}$ can be treated as nearly constant. Which the values obtained with nonexponential model for the delay below $100 \mu \mathrm{s}$, the $k_{T}^{\mathrm{df} \text {,ne }}$ grow up with the increase of $t_{0}$. This course we interpret as anti-Smoluchowski behavior. The values of $k_{T}^{\mathrm{df}, \text { ne }}$ are scattered around the constant value for the delay $100 \mu \mathrm{s}<t_{0}<\sim 5.5 \mathrm{~ms}$. They are smaller than the average value of $k_{T}^{\mathrm{df}, \mathrm{e}}$ calculated for the delay larger than $1 \mathrm{~ms}$. For $t_{0}$ above $5.5 \mathrm{~ms}$ the values of the first-order rate constant obtained from nonexponential model tend to the values obtained for exponential fit. The values of the first-order rate constant obtained from evaluation of phosphorescence decays are smaller than that evaluated from the delayed fluorescence. In the range $\sim 100 \mu \mathrm{s}--5.5 \mathrm{~ms}$ the first-order rate constant calculated with different approaches, with exponential and nonexponential models, can be arranged in ascending order $k_{T}^{\mathrm{ph} \text {,ne }}<k_{T}^{\mathrm{ph}, \mathrm{e}}<k_{T}^{\mathrm{df} \text {,ne }}<k_{T}^{\mathrm{df}, \mathrm{e}}$. Figure 2(b) presents the value of the indicator of nonexponential behavior $P_{4}$ (circles) as a function of the $t_{0}$. The course of this parameter versus the delay corresponds to the behavior of $k_{T}^{\mathrm{df} \text {,ne }}$. For the delay below $\sim 100 \mu$ s (the first experimental point only) the value decreases with the increase of the $t_{0}$. For the delay from the range $\sim 100 \mu \mathrm{s}-5.5 \mathrm{~ms}$ the values are scattered around the constant value $\approx 0.025$. For larger $t_{0}$ the coefficient $P_{4}$ tends to zero. Two inserts present: the $\chi^{2}$ function (right picture) and the parameter $P_{2}=R_{A} /\left(\pi D_{T}\right)^{1 / 2}$ (left picture) obtained from the temporal behavior of the delayed fluorescence. The values of $\chi^{2}$ in the case of nonexponential fit (circles) are equal to 1 with the exception of the first point, whereas the same parameter obtained for exponential fit (squares) decreases with the increase of $t_{0}$ within the range of delay $t_{0}$ below a few ms. For larger delay the values of $\chi^{2}$ obtained from both types of model: exponential and nonexponential are equal. The $P_{2}$ parameter calculated from nonexponential model (circles) has the values scattered around constant $\approx 0.7 \mathrm{~s}^{1 / 2}$ for $t_{0}$ within the range from $\sim 100 \mu$ s to $\sim 5.5 \mathrm{~ms}$. For smaller delay $P_{2}$ increases with the increase of $t_{0}$. For the delay above $5.5 \mathrm{~ms}$ the values of $P_{2}$ have large standard deviation $\Delta P_{2}$ and are spread over large range of values. The values of $P_{2}$ obtained from exponential fit (squares) grow up with the increase of $t_{0}$ up to about $10 \mathrm{~ms}$. The above description of the results can be summarized as follows: at the constant temperature there is the range of $t_{0}$ where $P_{2}, k_{T}^{\mathrm{df} \text {,ne }}$ and $P_{4}$ obtained from nonexponential fit is scattered around the constant.

The parameters obtained for the excitation with the "short pulse" have very similar dependence on the delay $t_{0}$ in comparison with data obtained from the middle excitation pulse. The example of experimental data (measured at $150 \mathrm{~K}$ ) analysis is described below. The values of $k_{T}^{\mathrm{df}, \mathrm{e}}$ can be treated as a nearly constant for the delay $t_{0}$ larger than about $1 \mathrm{~ms}$. 
For $t_{0}<\sim 1 \mathrm{~ms} ; k_{T}^{\mathrm{df}, \mathrm{e}}$ decreases with the increase of $t_{0}$. For $t_{0}<0.5 \mathrm{~ms}$ the increase of the value of the first-order rate constant $k_{T}^{\mathrm{df} \text {,ne }}$ with the increase of the delay is observed. $k_{T}^{\mathrm{df} \text {,ne }}$ can be treated as constant for $t_{0}$ from the range from $\sim 0.5 \mathrm{~ms}$ to $\sim 5 \mathrm{~ms}$. In the case of the delay longer than $\sim 5 \mathrm{~ms}$ the values of $k_{T}^{\mathrm{df} \text {,ne }}$ are much more scattered in comparison with that from the range $\sim 0.5 \mathrm{~ms}-\sim 5 \mathrm{~ms}$. Also the standard deviation $\Delta k_{T}^{\mathrm{df}, \text { ne }}$ increases with the increase of the delay. This increase is significant for $t_{0}>\sim 5 \mathrm{~ms}$. This behavior reflects the decreasing contribution of the short-time effect to the kinetics of TTA with the increase of $t_{0}$. In the range of $t_{0}$ where $k_{T}^{\mathrm{df} \text {,ne }}$ can be treated as constant the values of the firstorder rate constant obtained from different measurements (delayed fluorescence and phosphorescence) and different models (exponential and nonexponential) can be placed in the similar row as for excitation with the "middle pulse": $k_{T}^{\mathrm{ph}, \mathrm{ne}} \leq k_{T}^{\mathrm{df} \text {,ne }}<k_{T}^{\mathrm{ph}, \mathrm{e}}<k_{T}^{\mathrm{df}, \mathrm{e}}$. The course of indicator of nonexponential behavior $P_{4}$ corresponds to that of $k_{T}^{\mathrm{df} \text {,ne }}$. For the delay below $\sim 0.5 \mathrm{~ms}$ the value of the parameter decreases with the increase of $t_{0}$. In the range from $\sim 0.5 \mathrm{~ms}$ to $\sim 4.5 \mathrm{~ms}-\sim 5 \mathrm{~ms}$ the value can be treated as constant within the accuracy of the measurement and the fitting procedure. For larger values of the delay $P_{4}$ is scattered and its accuracy $\Delta P_{4}$ increases with the increase of the $t_{0}$. The values of $P_{2}$ obtained from exponential model increase with the delay up to $t_{0} \approx 8 \mathrm{~ms}$. In the case of the nonexponential fit the value of $P_{2}$ can be treated as constant for the delay from the range $\sim 0.5 \mathrm{~ms}-\sim 5 \mathrm{~ms}$. Outside this range the parameter $P_{2}$ has different course:

(i) it increases with the increase of $t_{0}$, for the $t_{0}$ $<\sim 0.5 \mathrm{~ms}$;

(ii) in the case of $t_{0}>\sim 0.5 \mathrm{~ms}$ its scattering and standard deviation grows up with the delay.

For $t_{0}$ below $4.5 \mathrm{~ms}-5 \mathrm{~ms}$ the values of $\chi^{2}$ calculated from exponential model are larger than the values obtained for nonexponential fit. For the longer delays $\left(t_{0}>\sim 0.5 \mathrm{~ms}\right)$ the values of $\chi^{2}$ are equal for both models.

Since the kinetics of delayed fluorescence depends on the mobility of molecules in $T_{1}$ state the differences between exponential and nonexponential models of TTA should be much larger in the case of delayed fluorescence than in the case of phosphorescence. Generally the temperature range of the study can be divided into two parts:

(i) in the first one the exponential model gives reasonable description of the kinetics of TTA;

(ii) in the other one the application of nonexponential model is necessary in order to obtain reasonable values of the kinetic parameters.

The temperature where the kinetic model has to be change from exponential to nonexponential will be called transition temperature.

In Figure 3 there is presented the first-order rate constant of the molecules in the $T_{1}$ state as dependent on temperature. Squares represent the data obtained from the fitting procedure of the delayed fluorescence with application of the

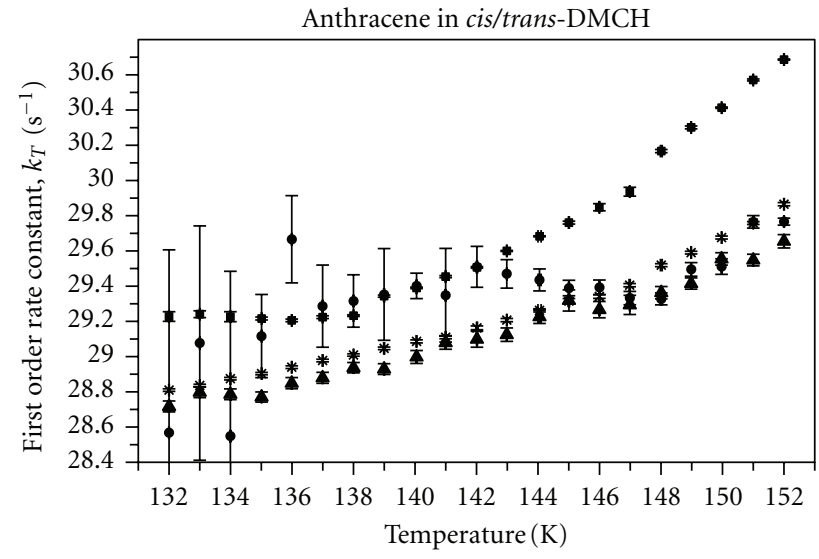

(a)

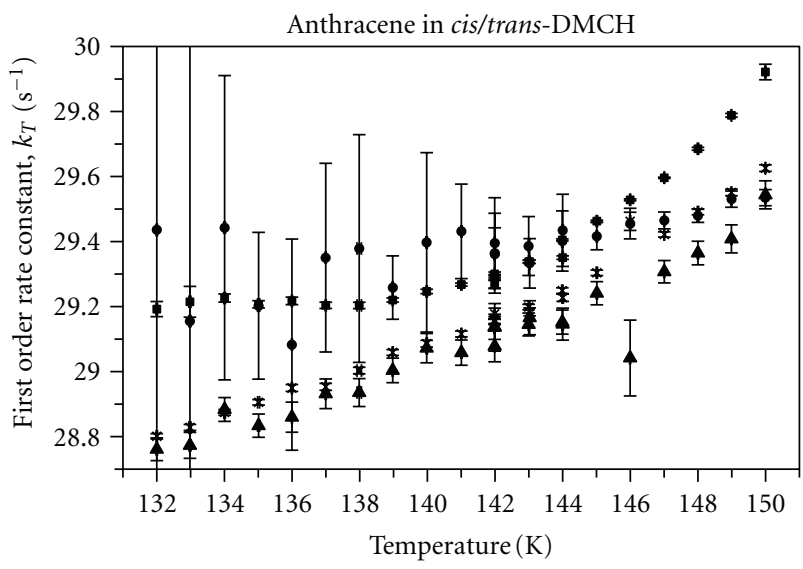

- Delayed fluorescence, exponential fit

- Delayed fluorescence, nonexponential fit

$\times$ Phosphorescence, exponential fit

$\Delta$ Phosphorescence, nonexponential fit

(b)

FIGURE 3: First-order rate constant as a function of temperature. Part (a): measurements performed with middle excitation pulse (dye laser pumped excimer laser), part (b) measurements in the case of excitation with short pulse (dye laser pumped nitrogen laser). Results of following measurements and fits are compared: exponential fit of the delayed fluorescence (squares), nonexponential fit of the delayed fluorescence (circles), exponential fit of the phosphorescence (crosses), and nonexponential fit of the phosphorescence (triangles). All fits were performed for intuitively modified model.

exponential model $-k_{T}^{\mathrm{df}, \mathrm{e}}$. Stars represent the result of the fitting of the exponential model to phosphorescence decays$k_{T}^{\text {ph,e }}$. The values of the first-order rate constant obtained from nonexponential model are represented by: circlesdelayed fluorescence $\left(k_{T}^{\mathrm{df}, n e}\right)$ and triangles — phosphorescence $\left(k_{T}^{\text {ph,ne }}\right)$. Part (a) presents the data measured when molecules are excited with the middle pulse, part (b) for excitation with the short one. The ordinate of both parts of the figure is expanded: only the range between $28.6 \mathrm{~s}^{-1}$ and $30.8 \mathrm{~s}^{-1}$ is shown. This is done in order to present small changes of the first-order rate constant obtained from different approaches. 
The behavior of the parameter in both parts of Figure 3 is similar.

The values of the first-order rate constant obtained from phosphorescence with application of both models are not very different from each other. Generally, the values of $k_{T}^{\mathrm{ph}, \text { ne }}$ are only slightly smaller in comparison with those of $k_{T}^{\mathrm{ph}, \mathrm{e}}$. There is one exception. The value of $k_{T}^{\mathrm{ph}, \mathrm{ne}}$ obtained from fit of the data measured at $146 \mathrm{~K}$ and with short excitation pulse is placed significantly below the course of the first-order rate constant obtained for other temperatures (Figure $3(\mathrm{~b})$ ). Also the accuracy of $k_{T}^{\mathrm{ph}, \mathrm{ne}}$ at $146 \mathrm{~K}$ is significantly larger in comparison with the values obtained for measurements performed at other temperatures. It suggests that in these conditions the fit of the nonexponential model to the phosphorescence decay gives larger uncertainty in comparison with other temperatures. Since this temperature can be treated as a transition temperature between the ranges of applicability of both models (exponential and nonexponential) the uncertainty may result from the large number of parameters in the nonexponential model. Parameters in the nonexponential model like indicator of nonexponential behavior has small values and standard deviation almost equal to $100 \%$ of the value (see Figure 4 and its discussion). Small values and large standard deviation of this parameter influence the values of other parameters.

Since the middle excitation pulse has significantly larger energy than the short one the transition temperature should be different for different types of excitation pulses. The temperature range of the applicability of exponential model is defined as a temperature region where the values of the first-order rate constant calculated from delayed fluorescence and phosphorescence build two almost parallel lines [22]. This range is as follows:

(i) $138 \mathrm{~K}-142 \mathrm{~K}$ for middle excitation pulse;

(ii) $138 \mathrm{~K}-146 \mathrm{~K}$ in the case of the short excitation pulse.

The temperature dependence of the $k_{T}^{\mathrm{df}, \mathrm{e}}$ below the transition temperature is the same as that obtained for intuitively modified model [22]. Below $138 \mathrm{~K}$ the differences between $k_{T}^{\mathrm{df}, \mathrm{e}}$ and $k_{T}^{\mathrm{ph}}$ increase with the decreasing of the temperature. The reasons for this behavior were suggested in the previous papers $[19,22]$. For the temperature values above the transition temperature the difference between $k_{T}^{\mathrm{df}, \mathrm{e}}$ and $k_{T}^{\mathrm{ph}}$ increases with the increase of the temperature. The temperature dependence of the $k_{T}^{\mathrm{df} \text {,ne }}$ shows the advantage of the nonexponential model in the temperature range above the transition temperature. In this temperature range the values of the $k_{T}^{\mathrm{df} \text {,ne }}$ are much closer to $k_{T}^{\mathrm{ph}}$ than the values of $k_{T}^{\mathrm{df}, \mathrm{e}}$. For the middle excitation pulse the values of $k_{T}^{\mathrm{df} \text {,ne }}$ are placed between the values of $k_{T}^{\mathrm{df}, \mathrm{e}}$ and $k_{T}^{\mathrm{ph}}$ in the temperature range $143 \mathrm{~K}-145 \mathrm{~K}$. For the values of the temperature higher than $145 \mathrm{~K}$ the values of $k_{T}^{\mathrm{df} \text {,ne }}$ are placed very close to the $k_{T}^{\mathrm{ph}, \mathrm{ne}}$.

The values of $k_{T}^{\mathrm{df} \text {,ne }}$ obtained for the temperature below the transition temperature have much larger standard deviation $\left(\Delta k_{T}^{\mathrm{df} \text {,ne }}\right)$ in comparison with the values obtained from

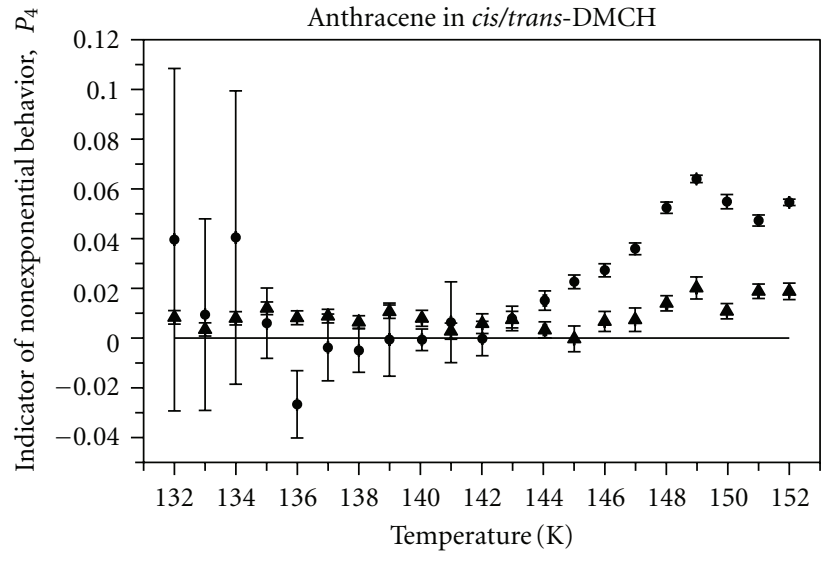

(a)

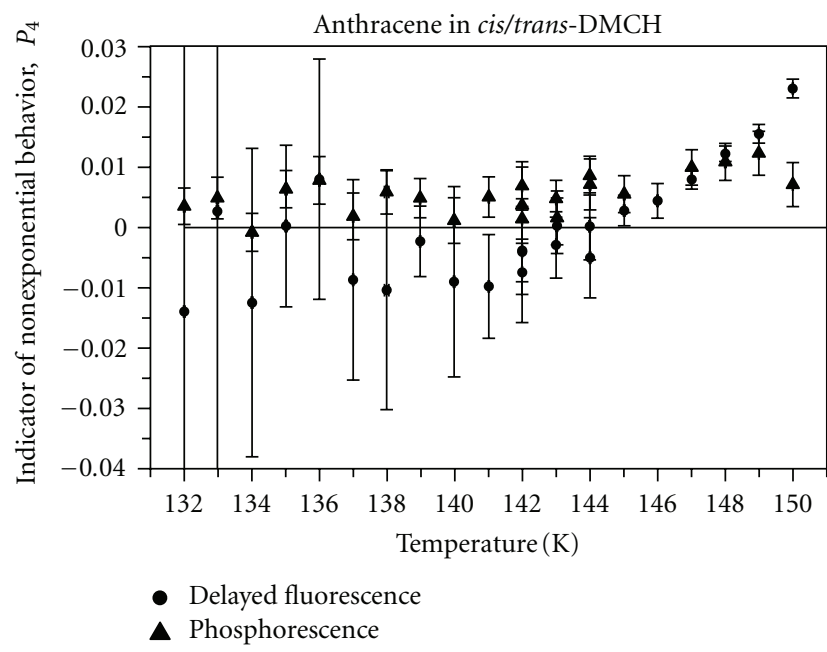

(b)

FIGURE 4: Indicator of nonexponential behavior as dependent on the temperature measured for excitation with the middle pulsepart (a) and short pulse-part (b). Comparison of the data obtained from the fit of the delayed fluorescence (circles) and phosphorescence (triangles).

decays measured above the transition temperature. Also the increase of $\Delta k_{T}^{\mathrm{dfne}}$ with the decrease of the temperature is observed within the range below the transition temperature. This suggests that the nonexponential model may produce some artificial effects due to too large number of parameters if it is applied for the experimental conditions where the nonexponential effects are negligible. In this case the parameter describing nonexponential behavior has small value and the uncertainty over $100 \%$ influences the values of other parameters and their standard deviations.

In Figure 4, there is presented the indicator of nonexponential behavior $-P_{4}$. The data for middle excitation pulse are shown in part (a), for short excitation pulse-in part (b). The circles represent the data obtained from fitting procedure applied to the decays of delayed fluorescence, triangles-phosphorescence. The temperature dependence of 
the parameter $P_{4}$ confirms the conclusion that the nonexponential model offers better description of the kinetics for the temperatures higher than the transition temperature. Starting from $146 \mathrm{~K}$ the value of $P_{4}^{\mathrm{df}}$ increases with the increase of the temperature. In the case of the middle excitation pulse (Figure 4(a)) the values of the indicator of nonexponential behavior calculated from delayed fluorescence $\left(P_{4}^{\mathrm{df}}\right)$ increase with the increase of the temperature within the temperature range $142 \mathrm{~K}-149 \mathrm{~K}$ and reaches the value of $\sim 0.06$ at $149 \mathrm{~K}$. In the temperature range $149 \mathrm{~K}-152 \mathrm{~K}$ the values of $P_{4}^{\mathrm{df}}$ are scattered around the constant. Below the transition temperature the values of $P_{4}^{\mathrm{df}}$ are scattered around zero and the standard deviation $\Delta P_{4}^{d f}$ is generally larger than $\left|P_{4}^{\text {df }}\right|$. The data obtained from the excitation with the short pulse show very similar behavior. Since the energy of short pulses was much smaller the transition temperature is higher and equals $146 \mathrm{~K}$. Also the range of changes of $P_{4}^{\mathrm{df}}$ is smaller in comparison with the data obtained for middle excitation pulse. The values of $P_{4}^{\mathrm{df}}$ increase with the increase of the temperature within the temperature range from $146 \mathrm{~K}$ to $150 \mathrm{~K}$. The maximum of $P_{4}^{\mathrm{df}}$ equals to $\sim 0.02$ at $150 \mathrm{~K}$. Below the transition temperature the behavior of the indicator of nonexponential behavior is the same as for the middle excitation pulse. The values of the indicator of nonexponential behavior calculated from the phosphorescence $\left(P_{4}^{\mathrm{ph}}\right)$ are generally slightly larger than zero in the whole temperature range of investigation. The tendency that the values of $P_{4}$ grow up with the increase of the temperature in the range above the transition temperature is observed only for the delayed fluorescence. The reason for this behavior is that the contribution of the diffusion-controlled process to the phosphorescence decay is much smaller than to the delayed fluorescence. In turn: the contribution of nonexponential effect should be here much smaller in comparison with the delayed fluorescence.

Comparison of the kinetic parameters obtained from intuitive and non-Fickian models is presented in Figures 5 and 6. In each figure part (a) presents the data obtained for intuitive treatment, part (b)-for non-Fickian model. In Figure 5 the first-order rate constant is shown as a function of temperature. The data were obtained for middle excitation pulse. In the case of application of the intuitive model to the experimental data measured for middle excitation pulse the kinetics of diffusion-controlled TTA is well described within the exponential approximation for the temperature below $142 \mathrm{~K}$. For the temperature between $142 \mathrm{~K}$ (transition temperature) and $149 \mathrm{~K}$ the values of the first-order rate constant obtained from (20) have the same course as presented in Figure 3(a). Above $149 \mathrm{~K}$ the approximation used in (20) do not give reasonable fit of the model to the experimental decay. In Figure 5(a) there are also presented the values of the first-order rate constant obtained from the fit of phosphorescence. The comparison of $k_{T}^{\mathrm{df} \text {,ne }}$ obtained from (20) with $k_{T}^{\mathrm{df}, \mathrm{e}}$ and $k_{T}^{\mathrm{ph}}$, shows that simplified nonexponential model defined by (20) gives the same results as model defined by (17).

In Figure 5(b) the temperature dependence of the firstorder rate constant calculated from the nonexponential

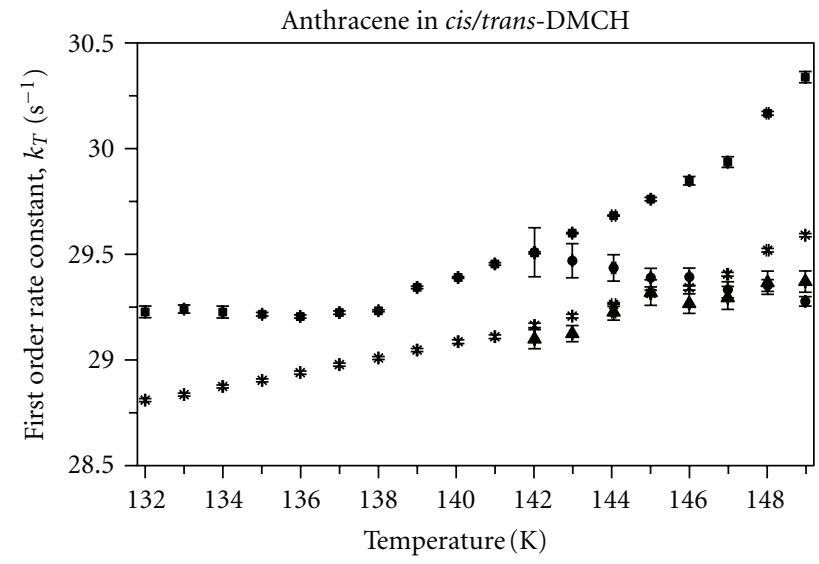

(a)

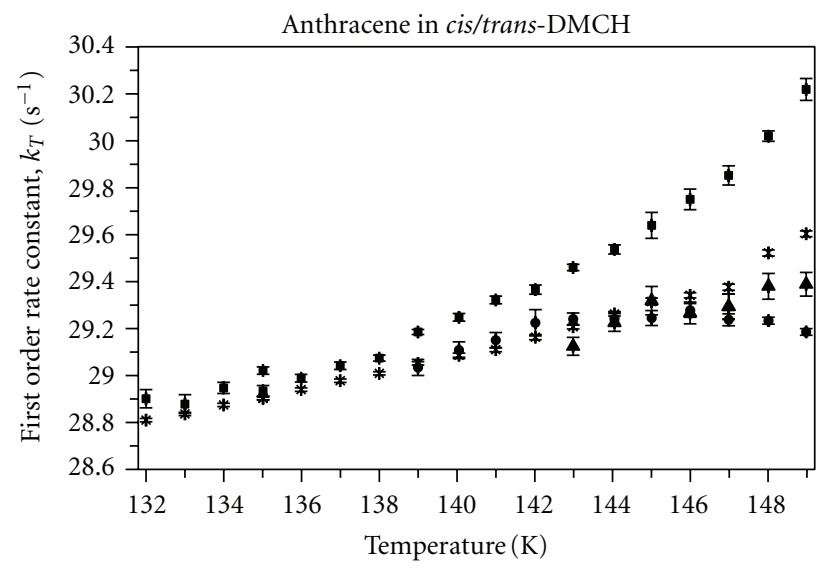

- Delayed fluorescence, exponential fit

- Delayed fluorescence, nonexponential fit

$\times$ Phosphorescence, exponential fit

A Phosphorescence, nonexponential fit

(b)

Figure 5: Comparison of the values of the first-order rate constant calculated with intuitive (a) and non-Fickian (b) models. The values of the parameter are presented as dependent on temperature. The measurements were performed for so-called middle excitation pulse.

version of non-Fickian model is compared with the data obtained for this model within exponential approximation. The nonexponential modification introduces no significant correction to the values of the first-order rate constant calculated from phosphorescence decays below $146 \mathrm{~K}$. For higher temperatures the values of $k_{T}^{\text {ph,ne }}$ have the tendency to be smaller than $k_{T}^{\mathrm{ph}, \mathrm{e}}$. The values of the first-order rate constant calculated from delayed fluorescence within non-Fickian exponential model are almost equal to that calculated from phosphorescence in the temperature range below $138 \mathrm{~K}$. For higher temperatures the difference between $k_{T}^{\mathrm{df}, \mathrm{e}}$ and $k_{T}^{\mathrm{ph}, \mathrm{e}}$ is observed: $k_{T}^{\mathrm{df}, \mathrm{e}}>k_{T}^{\mathrm{ph}, \mathrm{e}}$. This difference increases with the increase of the temperature. Application of nonexponential model extends the range of coincidence of values of $k_{T}^{\mathrm{df} \text {,ne }}$ and $k_{T}^{\text {ph,e }}$ up to about $146 \mathrm{~K}$. For higher temperatures the values 


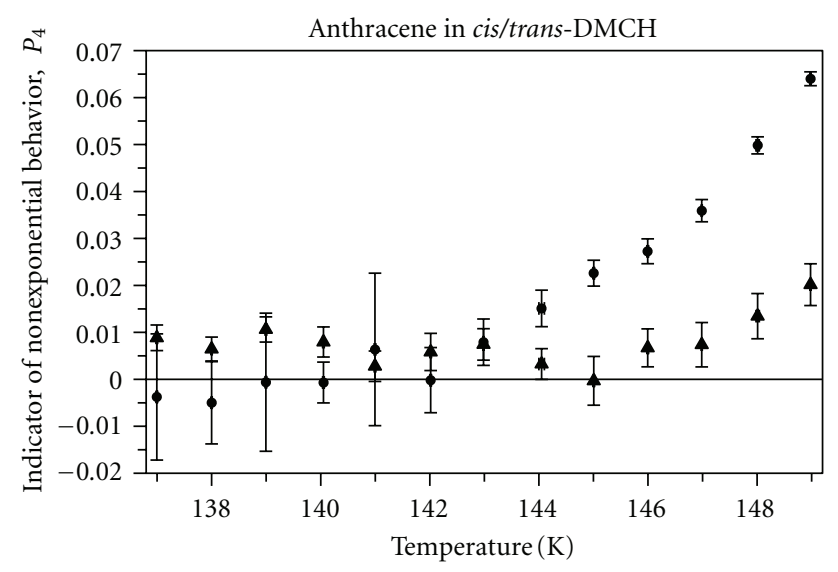

(a)

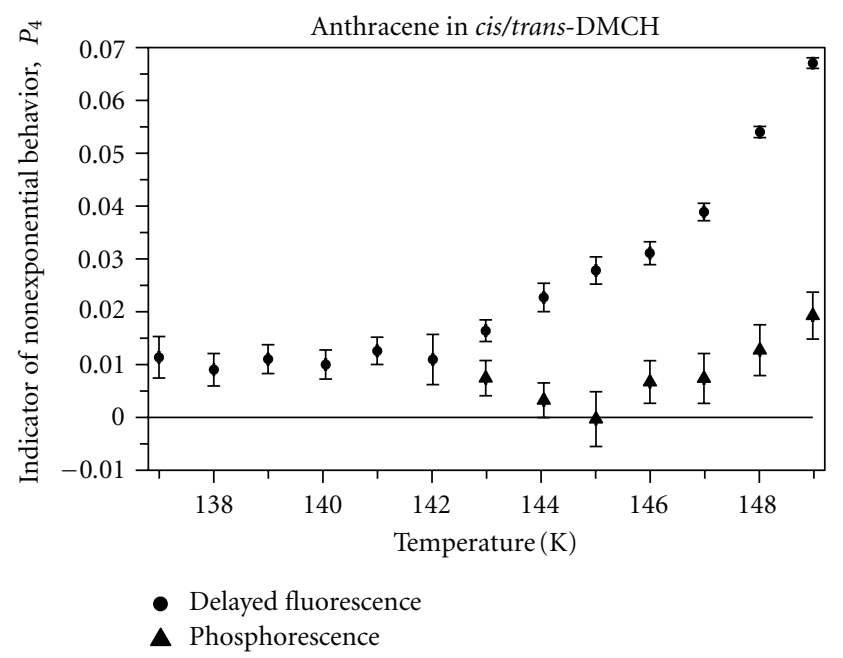

(b)

Figure 6: Comparison of the values of the indicator of nonexponential behavior calculated with intuitive (a) and non-Fickian (b) models. The values of the parameter are presented as dependent on temperature. The measurements were performed for socalled middle excitation pulse.

of $k_{T}^{\mathrm{df} \text {,ne }}$ are smaller than $k_{T}^{\mathrm{ph}, \mathrm{e}}$. This difference increases with the increase of the temperature.

The behavior of the first-order rate constant for short excitation pulse is similar to that presented for middle excitation pulse. The differences between exponential and nonexponential models are smaller because the energy of excitation pulse was smaller in comparison with the energy of middle excitation pulse. The lower energy of excitation pulse results in lower concentration of molecules in the triplet state.

The temperature dependence of the indicator of nonexponential behavior $\left(P_{4}\right)$ for middle excitation pulse is presented in Figure 6. As in the case of the first-order rate constant part (a) of this figure concerns the data obtained from intuitive model, part (b)_from non-Fickian model. The values of the parameter $P_{4}$ calculated within intuitive model from the delayed fluorescence are equal to zero within the accuracy of the measurement and the evaluation procedure for the temperature below $142 \mathrm{~K}$. For the temperature above $142 \mathrm{~K}$ the values of the indicator of nonexponential behavior increase with the increase of the temperature up to about 0.06 for $149 \mathrm{~K}$. In the case of the temperature values larger than $149 \mathrm{~K}$ the fit of (21) to the decays of the delayed fluorescence do not give stable result. The values calculated from phosphorescence grow up with the temperature in the range from $145 \mathrm{~K}$ to $149 \mathrm{~K}$. In the temperature below $145 \mathrm{~K}$ the values of $P_{4}$ (calculated from phosphorescence) are scattered between 0 and 0.01. In the case of non-Fickian model (Figure 6(b)) the values of $P_{4}$ calculated from delayed fluorescence are scattered around 0.01 for the temperature below $142 \mathrm{~K}$. Above this temperature the values of the indicator of nonexponential behavior increase with the increase of temperature. The largest value of $P_{4}$ equals to about 0.06 for $149 \mathrm{~K}$. The behavior of the indicator of nonexponential behavior observed for short excitation pulse is qualitatively the same as for middle pulse. Here however the transition temperature equals to $145 \mathrm{~K}$ instead of $142 \mathrm{~K}$. Also the largest value of $P_{4}$ reached at $149 \mathrm{~K}$ equals to 0.02 instead of 0.06 reached at the same temperature for middle excitation pulse. The reason for this difference is, as mentioned above, the lower energy of the exciting pulse. Pulses having lower excitation energy generate lower concentration of the molecules in the $T_{1}$ state. This implies that nonexponential behavior gives measurable component to the decay in the system with higher mobility of the molecules, that means for the conditions where the diffusion coefficient is larger.

To sum up, the introducing of the nonexponential model offers better description of the kinetics within this temperature range in which the combination of the energy of excitation pulse and the mobility of molecules results in significant deviation from the exponential model. Both models: the intuitive and non-Fickian has their limits. The intuitive model has the tendency to overestimate the first-order rate constant calculated from the delayed fluorescence in comparison with the value obtained from the phosphorescence. The course of the first-order rate constant calculated from the delayed fluorescence with nonFickian model does not show this systematic difference. At the other side the non-Fickian model gives a good fit to the experimental decay within the smaller range of the temperature.

The indicator of nonexponential behavior obtained from intuitive model equals to zero within the temperature range where the exponential model offers good description. In the case of non-Fickian model this parameter tends to 0.01 instead of zero with the standard deviation significantly smaller than $100 \%$ in the temperature range where the exponential approach offers good description of the kinetics.

The most important conclusion coming out from the paper is that the nonexponential approach significantly enhanced the range of experimental conditions where the kinetics of diffusion-controlled TTA has reasonable description. The indicator of nonexponential behavior-parameter 
$P_{4}$ should be $<0.01$ if the exponential approach provides reasonable description of the kinetics. The nonexponential model allowed the use of the model up to values of $P_{4}$ about six times larger $\left(P_{4}^{\max } \approx 0.06\right)$.

The annihilation radius can be calculated from equation for $P_{2}=R_{\mathrm{A}} /\left(\pi D_{T}\right)^{1 / 2}$ parameter. This evaluation requires the value of mutual diffusion coefficient $D_{T}$. The values of diffusion coefficient measured in other solvents and extrapolated to experimental conditions used here give reasonable values of annihilation radius $R_{A}$ at least in the temperature $136 \mathrm{~K}-149 \mathrm{~K}$ [22]. However, the values of $D_{T}$ extrapolated from other solvents do not take into account the specific features of the solvent mixture used in this work. Sophisticated proof of the model requires the values diffusion coefficient measured at the same experimental conditions as presented here for kinetic study. Values of $D_{T}$ can be calculated from decays of delayed fluorescence obtained from measurements with spatially periodic excitation. This kind of excitation requires in turn further extension of the nonexponential approach. The introducing of the concept of joined action of the diffusion and the first-order decay to the triplet-triplet annihilation kinetics in the case of spatially periodic excitation will be the subject of subsequent work.

\section{Acknowledgments}

The authors thank Professor Jürgen Troe (Max-Planck Institute, Göttingen, Germany) for generous support of the research project. The discussion with Professor K. Rotkiewicz (Institute of Physical Chemistry PAS, Warsaw, Poland) was very fruitful for preparation of this manuscript. The support of stay in Göttingen (Germany) of one of the authors (P. B.) by Max-Planck-Gesellschaft (from 1.10.2000 to 30.09.2001) and by Alexander von Humboldt-Foundation (from 1.10.2001 to 31.12.2002) is gratefully acknowledged. Dr B. Nickel passed away 27/01/2002.

\section{References}

[1] A. M. North, "Diffusion-controlled reactions," Quarterly Reviews of the Chemical Society, vol. 20, pp. 421-440, 1966.

[2] M. Sikorski, E. Krystkowiak, and R. P. Steer, "The kinetics of fast fluorescence quenching processes," Journal of Photochemistry and Photobiology A, vol. 117, no. 1, pp. 1-16, 1998.

[3] E. Krystkowiak and A. Maciejewski, "Kinetics of fluorescence quenching of $\mathrm{S}_{2}$-thioketones studied by the SmoluchowskiCollins-Kimball model: standard systems," Journal of Chemical Physics, vol. 117, no. 5, pp. 2246-2254, 2002.

[4] M. Litniewski and J. Gorecki, "Molecular dynamics tests of the Smoluchowski-Collins-Kimball model for fluorescence quenching of spherical molecules," Physical Chemistry Chemical Physics, vol. 6, no. 1, pp. 72-83, 2004.

[5] A. Morandeira, A. Fürstenberg, J.-C. Gumy, and E. Vauthey, "Fluorescence quenching in electron-donating solvents. 1. Influence of the solute-solvent interactions on the dynamics," Journal of Physical Chemistry A, vol. 107, no. 28, pp. 53755383, 2003.

[6] A. Morandeira, A. Fürstenberg, and E. Vauthey, "Fluorescence quenching in electron-donating solvents. 2. Solvent dependence and product dynamics," Journal of Physical Chemistry A, vol. 108, no. 40, pp. 8190-8200, 2004.
[7] K. M. Solntsev, D. Huppert, N. Agmon, and L. M. Tolbert, "Photochemistry of "super" photoacids. 2. Excited-state proton transfer in methanol/water mixtures," Journal of Physical Chemistry A, vol. 104, no. 19, pp. 4658-4669, 2000.

[8] L. M. Tolbert and K. M. Solntsev, "Excited-state proton transfer: from constrained systems to "super" photoacids to superfast proton transfer," Accounts of Chemical Research, vol. 35, no. 1, pp. 19-27, 2002.

[9] E. Pines and D. Huppert, "Observation of geminate recombination in excited state proton transfer," Journal of Chemical Physics, vol. 84, no. 6, pp. 3576-3577, 1986.

[10] N. Agmon, D. Huppert, A. Masad, and E. Pines, "Excitedstate proton transfer to methanol-water mixtures," Journal of Physical Chemistry, vol. 95, no. 25, pp. 10407-10413, 1991.

[11] E. Pines, D. Huppert, and N. Agmon, "Geminate recombination in excited-state proton transfer reactions: numerical solution of the Debye-Smoluchowski equation with backreaction and comparison with experimental results," Journal of Chemical Physics, vol. 88, no. 9, pp. 5620-5630, 1988.

[12] N. Agmon, E. Pines, and D. Huppert, "Geminate recombination in proton-transfer reactions. II. Comparison of diffusional and kinetic schemes," Journal of Chemical Physics, vol. 88, no. 9, pp. 5631-5638, 1988.

[13] I. V. Gopich, K. M. Solntsev, and N. Agmon, "Excitedstate reversible geminate reaction. I. Two different lifetimes," Journal of Chemical Physics, vol. 110, no. 4, pp. 2164-2174, 1999.

[14] N. Agmon, "Excited-state reversible geminate reaction. II. Contact geminate quenching," Journal of Chemical Physics, vol. 110, no. 4, pp. 2175-2180, 1999.

[15] K. M. Solntsev, D. Huppert, and N. Agmon, "Photochemistry of "super"-photoacids. Solvent effects," Journal of Physical Chemistry A, vol. 103, no. 35, pp. 6984-6997, 1999.

[16] E. Pines, B. Z. Magnes, M. J. Lang, and G. R. Fleming, "Direct measurement of intrinsic proton transfer rates in diffusioncontrolled reactions," Chemical Physics Letters, vol. 281, no. 46, pp. 413-420, 1997.

[17] M. von Smoluchowski, "Versuch einer mathematischen Theorie der Koagulationskinetik kolloider Lösungen," Zeitschrift für Physikalische Chemie, vol. 92, pp. 129-168, 1917.

[18] B. Nickel, P. Borowicz, A. A. Ruth, and J. Troe, "Application of Smoluchowski's generalized theory to the kinetics of triplettriplet annihilation of anthracene in viscous solution after long-pulse excitation," Physical Chemistry Chemical Physics, vol. 6 , no. 13, pp. 3350-3363, 2004.

[19] P. Borowicz and B. Nickel, "Triplet-triplet annihilation in viscous solutions as an example of non-Fickian diffusion," Journal of the Optical Society of America B, vol. 22, no. 2, pp. 315-322, 2005.

[20] F. C. Collins and G. E. Kimball, "Diffusion-controlled reaction rates," Journal of Colloid Science, vol. 4, no. 4, pp. 425-437, 1949.

[21] S. A. Rice, Comprehensive Chemical Kinetics: Diffusion-Limited Reactions, Elsevier, Amsterdam, The Netherlands, 1985.

[22] P. Borowicz and B. Nickel, "Application of high-accuracy timeresolved laser spectroscopy to the study of diffusion-controlled triplet-triplet annihilation," Opto-Electronics Review, vol. 12, no. 3, pp. 325-332, 2004.

[23] S. A. Rice, Comprehensive Chemical Kinetics: DiffusionLimitedReactions, Elsevier, Amsterdam, 1985.

[24] D. L. Dexter, "A theory of sensitized luminescence in solids," Journal of Chemical Physics, vol. 21, no. 5, pp. 836-850, 1953.

[25] H.-T. Chen and K.-C. Liu, "Analysis of non-Fickian diffusion problems in a composite medium," Computer Physics Communications, vol. 150, no. 1, pp. 31-42, 2003. 
[26] J. B. Birks, Photophysics of Aromatic Molecules, WileyInterscience, London, UK, 1970.

[27] B. Nickel, H. E. Wilhelm, and C. P. Jaensch, "Effect of the förster energy transfers $S_{1}+S_{1} \rightarrow S_{0}+S_{n}$ and $S_{1}+T_{1} \rightarrow$ $S_{0}+T_{m}$ on the time dependence of the delayed ffrom aromatic compounds: anti-smoluchowski and smoluchowski temporal behavior," Optics and Spectroscopy, vol. 83, no. 4, pp. 541-556, 1997. 


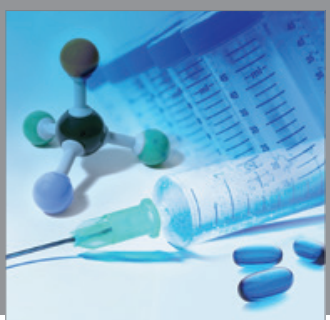

International Journal of

Medicinal Chemistry

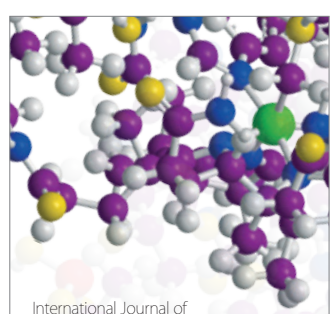

Carbohydrate Chemistry

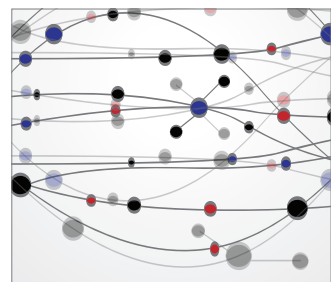

The Scientific World Journal
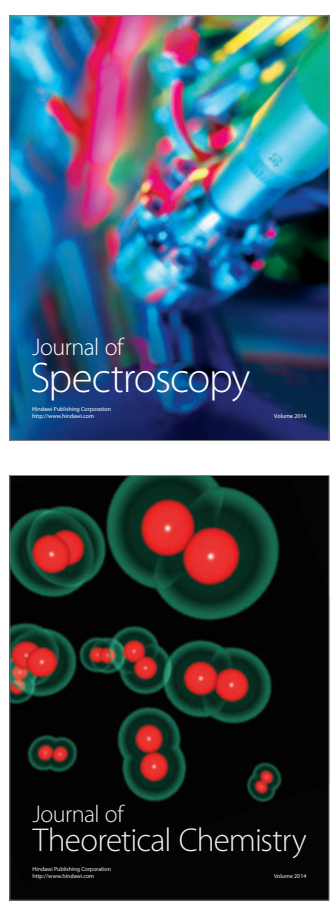
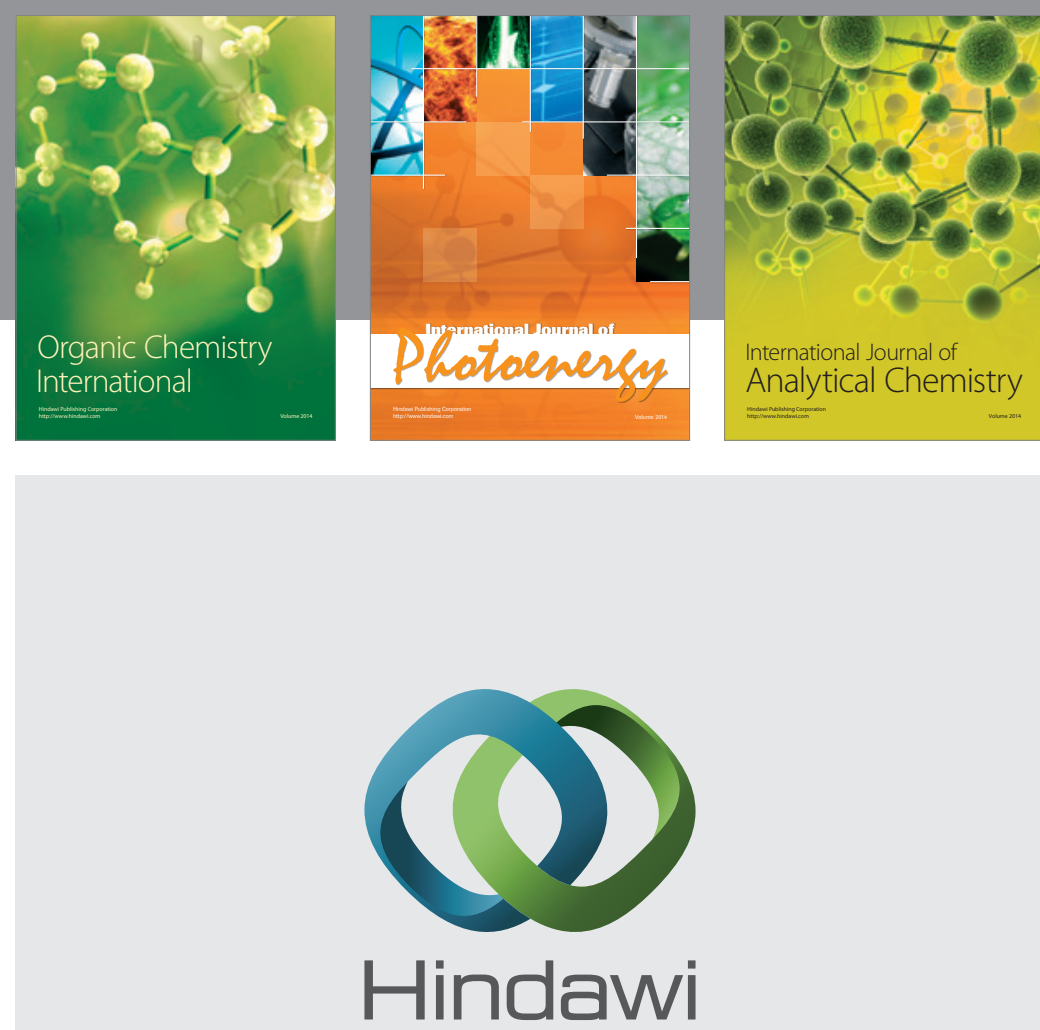

Submit your manuscripts at

http://www.hindawi.com
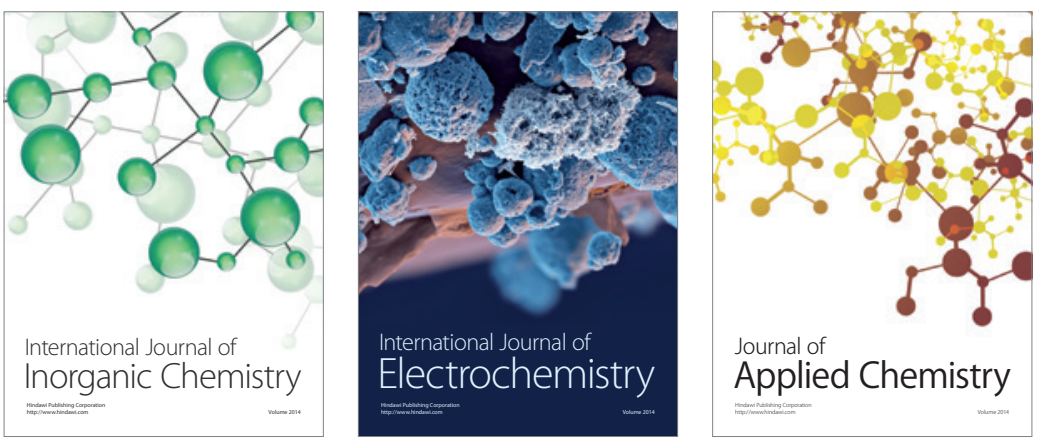

Journal of

Applied Chemistry
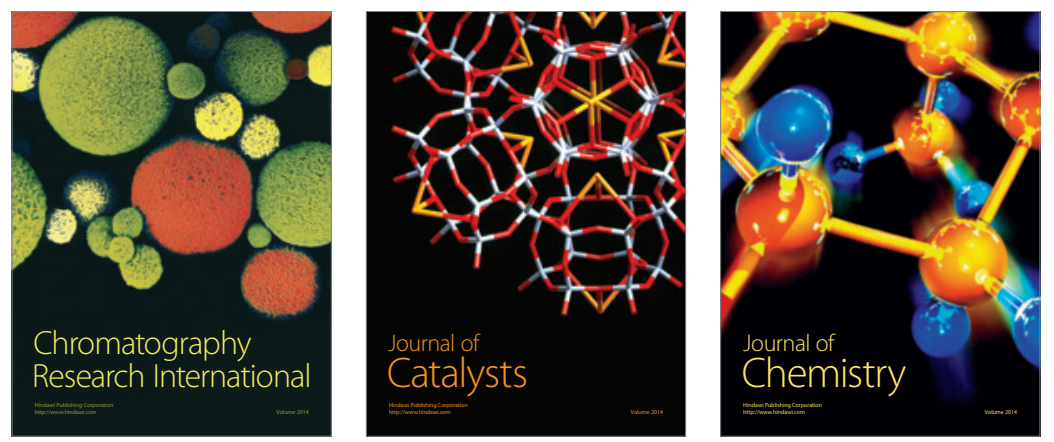
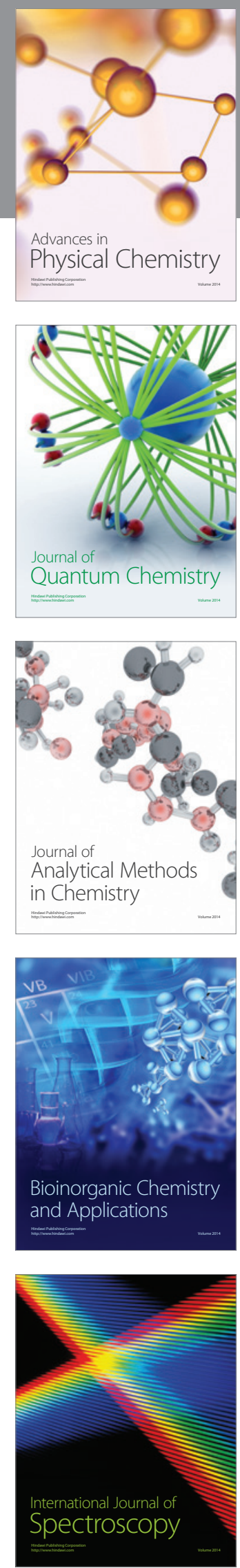